\title{
Dynamic computer-generated hologram displayed on a spatial light modulator for information processing
}

Pierre Ambs, Laurent Bigue, Christophe Stolz

Pierre Ambs, Laurent Bigue, Christophe Stolz, "Dynamic computer-generated hologram displayed on a spatial light modulator for information processing," Proc. SPIE 10296, 1999 Euro-American Workshop Optoelectronic Information Processing: A Critical Review, 1029608 (2 June 1999); doi: 10.1117/12.365918

EDent: Euro-American Workshop on Optoelectronic Information Processing, 1999, Colmar, France 


\title{
Dynamic computer generated hologram displayed on a spatial light modulator for information processing
}

\author{
Pierre Ambs, Laurent Bigué, Christophe Stolz \\ Université de Haute Alsace \\ Ecole Supérieure des Sciences Appliquées pour l'Ingénieur-Mulhouse \\ 12 rue des Frères Lumière, 68093 Mulhouse Cedex, France
}

\begin{abstract}
Many implementations of computer generated holograms (CGH's) or diffractive optical elements (DOE's) onto spatial light modulators (SLM's) have already been considered. In this paper, we first review the various types of SLM's available for DOE's and the implementations of DOE's onto SLM's already reported in the literature. Then we investigate the point in displaying DOE's onto SLM's that couple phase and amplitude modulations, such as twisted nematic-liquid crystal displays (TN-LCD's): we provide computer simulations as well as experimental results.
\end{abstract}

Keywords: diffractive optical element, computer generated hologram, spatial light

modulator, twisted nematic-liquid crystal display

\section{INTRODUCTION}

The diffractive Optical Elements (DOE's) have now reached a state of maturity. The expression DOE which is more general, is now often preferred to Computer Generated Hologram (CGH). Indeed, they can now be computed and encoded with powerful iterative algorithms, such as the Iterative Fourier Transform Algorithm (IFTA) ${ }^{1,2}$ or Direct Binary Search (DBS) ${ }^{3}$ which have been improved and optimized in order to combine high quality reconstruction with high diffraction efficiency. The fabrication process of DOE's is also better mastered, the association of e-beam lithography techniques with reactive ion beam etching allows the fabrication of multiple phase level elements with a feature size of about $1 \mu \mathrm{m}^{4}$. Cheaper fabrication techniques such as embossing also exist and allow the fabrication of good DOE's at low cost. The field of DOE's applications ${ }^{5}$ has expanded and includes diffractive lenses, beam shaping, structured light generation, optical interconnects.... Moreover, in order to add flexibility to the optical processors, the DOE's should be dynamic. A dynamic DOE must be displayed onto a Spatial Light Modulator (SLM) and it is only in the past few years that SLM's suitable for this application became available at a moderate price. Nevertheless, the SLM's are not perfect and many difficulties remain. In particular, their coding domain is limited and, depending on the technology used, several problems appear : the 
152 / Critical Reviews Vol. CR74

amplitude is coupled with the phase, the phase shift does not reach $2 \pi$, the spatial resolution is limited, the minimum feature size is still much larger than for fixed DOE's. Therefore, it is very important that the CGH encoding takes account of the SLM characteristics. Several methods have been proposed by different authors ${ }^{6-8}$; we propose in this paper a unified approach based on the use of Optimal Characteristics Curves (OCC). Applications of dynamic DOE's include programmable lenses, reconfigurable optical interconnects, dynamic display, structured light projection, reconfigurable filters....

In this paper, we will emphasize the use of commercially available, low cost SLM's because even if there are other types of SLM which are better adapted to dynamic DOE's, some are still in the state of laboratory research or are very expensive.

Section 2 presents an overview of the characteristics of the different SLM's available. A review of the implementations of CGH's onto SLM's is given in Section 3. Section 4 shows the use of OCC for mapping the CGH on the SLM coding domain. Section 5 provides an optical implementation that validates the simulations of Section 4 . The last section includes a summary and conclusions.

\section{SLM OVERVIEW}

SLM's can be classified into two categories, the ones addressed optically (OASLM) and the ones addressed electrically (EASLM). An EASLM consists of an electrical- optical interface and the optical output signal is pixelated. In the case of an OASLM, there is an optical write beam that controls the modulation of the read beam. The write beam can be coherent or incoherent. As far as DOE's applications are concerned, EASLM's are mostly used.

\subsection{Coding domains}

The coding domain of a SLM represents the range of complex values that can be reproduced. Ideally, we would like to code values in the full complex plane. But in fact, the values are limited. Since SLM's are passive devices that can only attenuate the beam that is passing through, the values will already be limited to the unity circle. Unfortunately, no existing SLM can address the whole unity circle; some are amplitude only modulators (Fig. $1 \mathrm{~b}$ ), some phase only modulators (Fig. $1 \mathrm{c}$ ) and in many cases the amplitude and the phase are coupled (Fig. li). 


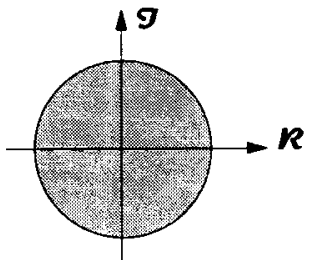

(a)

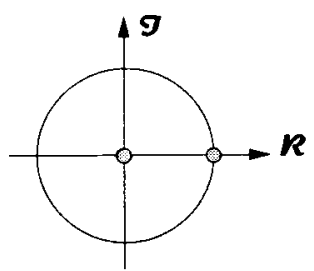

(d)

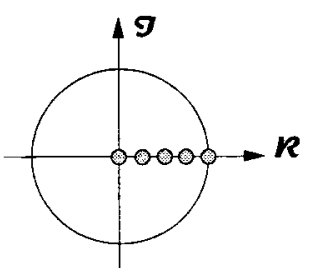

(g)

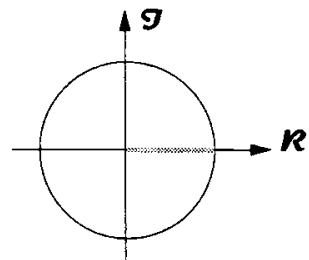

(b)

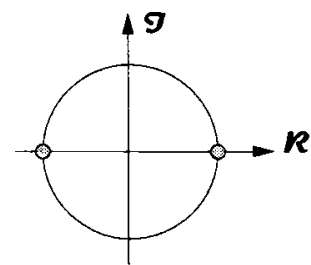

(e)

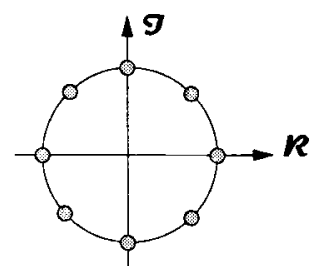

(h)

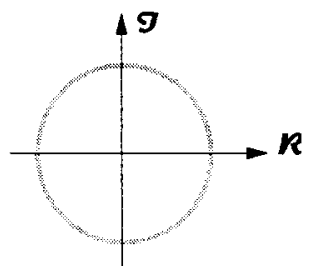

(c)

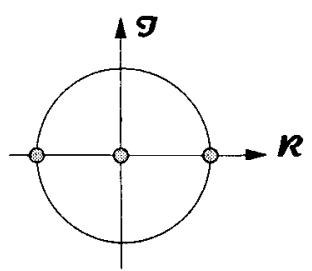

(f)

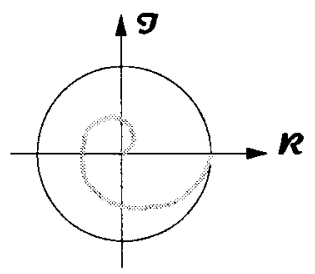

(i)

Fig. 1: Examples of coding domains: unity disk (a), pure amplitude (b), pure phase (c), amplitude only (d), pure binary phase (e), ternary phase (f), quantified amplitude only (g), quantified phase only (h), spiral (i)

\subsection{Different types of SLM's}

\subsubsection{Liquid crystal SLM's}

Most commercially available SLM's are liquid crystal devices. The liquid crystals show intermediate physical properties between liquids and solids ${ }^{9}$. They have a liquid aspect but present a structured molecular arrangement. There are different types of liquid crystals depending on the molecules' arrangement type.

The molecules are aligned parallel in a parallel aligned device (PAL-LCD) ${ }^{10}$. Such a cell behaves as a tunable retardation plate. PAL SLM's are commercialized by Hamamatsu. In a twisted nematic liquid crystal (TN-LC) ${ }^{9}$, the liquid crystal molecules are arranged in the form of a helix. The increase in the voltage applied to the cell changes the regularity of the twist. Therefore, the birefringence is modified, leading to a polarization and phase modulation. A polarizer placed at the output of the TN-LC SLM allows an amplitude modulation. According to the polarization direction of the beam illuminating the SLM and to the orientation of the output polarizer, different operating modes of the SLM are possible. These modes range from a near pure phase modulation mode to a near pure amplitude modulation mode.

In a ferro-electric liquid crystal (FLC) ${ }^{11}$ there is a bistable electro-optic effect which can give a binary phase or amplitude modulation. 


\subsubsection{Magneto-optic SLM's}

Magneto-optic (MO) SLM's are based on the Faraday effect ${ }^{12}$. They are used as bistable devices, but there is the possibility of using a third state to implement a ternary encoding ${ }^{13}$. Since the Faraday effect is weak, the light efficiency is very low (1-4\%), and therefore MO SLM's are now less and less used. They were produced by Litton Data Systems.

\subsubsection{Deformable Mirror Devices}

There are different types of deformable mirror devices (DMD). The DMD's with a deformable reflecting membrane modulate the phase ${ }^{14}$. Any complex value can be obtained by grouping several pixels together ${ }^{15}$. The DMD's currently commercialized by Texas Instruments ${ }^{16}$ are used in video projectors. Each pixel of the SLM consists of a mirror on a torsion that can have two positions, $\pm 10^{\circ}$, and therefore deflects the light in two directions.

\subsubsection{Other types of SLM's}

There are other types of SLM's, but they are not so easily available as the SLM's described previously. For example, multiple quantum wells (MQW $)^{17}$ devices consist of thin, alternate layers of quantum wells semiconductors. When appropriately polarized, these layers show an absorption variation and can therefore be used as modulators. In some conditions, they can be used as phase modulators ${ }^{18}$.

\subsection{Comparison}

\subsubsection{Coding domains}

\begin{tabular}{|c|c|c|c|c|c|c|}
\hline & TN-LC & PAL-LC & FLC/ELC & MO & DMD & MQW \\
\hline amplitude & 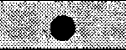 & & & & & 0 \\
\hline $\begin{array}{l}\text { quantized } \\
\text { amplitude }\end{array}$ & 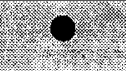 & & 0 & & & 0 \\
\hline \begin{tabular}{|l} 
binary \\
amplitude
\end{tabular} & 0 & & 0 & 0 & 0 & 0 \\
\hline phase & 0 & 0 & 0 & & 0 & 0 \\
\hline $\begin{array}{l}\text { quantized } \\
\text { phase }\end{array}$ & 0 & 0 & 0 & & 0 & 0 \\
\hline binary phase & 0 & 0 & 0 & 9 & 0 & 0 \\
\hline temary & - & & - & - & & \\
\hline $\begin{array}{l}\text { coupled } \\
\text { amplitude and } \\
\text { phase }\end{array}$ & 0 & & 0 & & & \\
\hline complex & - & & & & 0 & \\
\hline
\end{tabular}

Table 1: Summary of the coding domains of the different SLM's. The cells in shaded boxes mean that the implementation considered consists of an approximation of the desired coding domain. 
Table 1 presents a summary of the coding domains of each SLM described above. It can be noted that the TN-LC offers the largest range of coding domains even if the pure amplitude modulation is only partially reached.

\subsubsection{Operating Rate}

Fig. 2 shows that the SLM's can be classified into three categories according to their operating rate. TN-LC, PAL-LC and MO SLM's operate at video rate. FLC and DMD SLM's are faster (tens of kiloHertz) and MQW SLM's reach much higher rates. The figures given here include the addressing time in the case of an electrically addressed SLM.

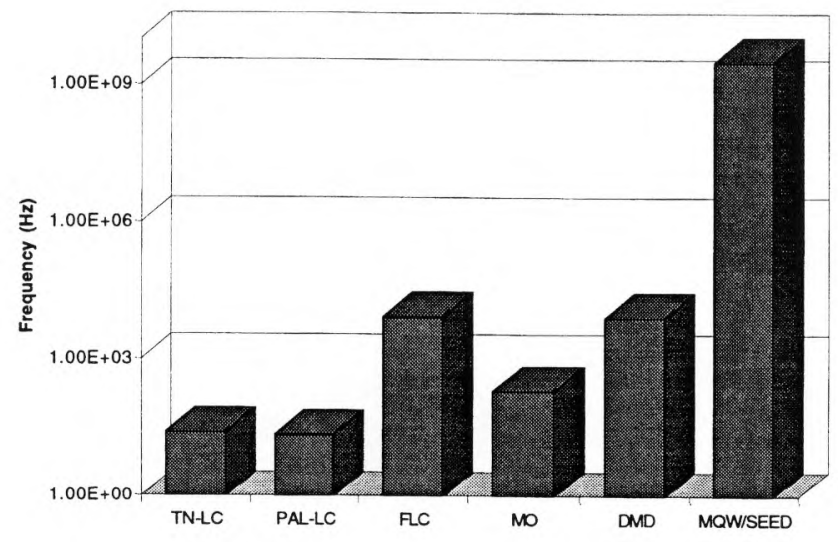

Fig. 2: Operating rate of various SLM's.

\subsubsection{Resolution}

The resolution of the various SLM's is approximately the same, but continuously increases with technological progress.

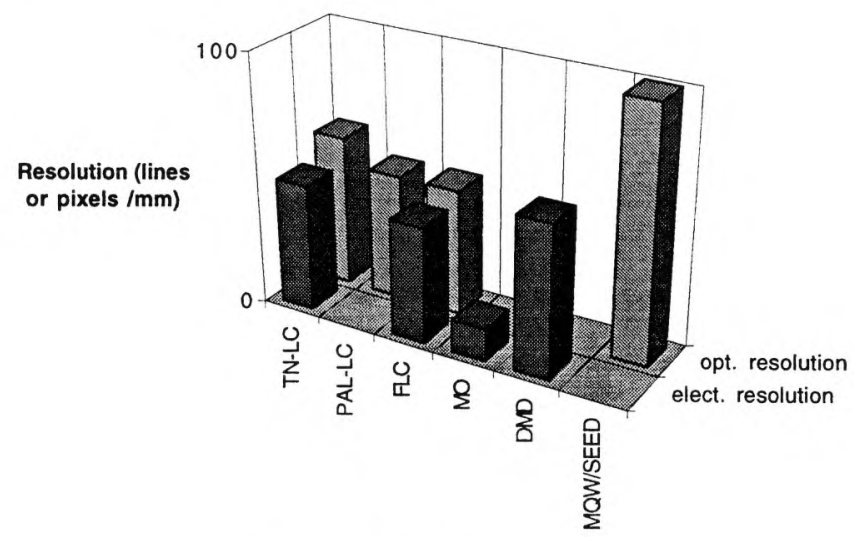

Fig. 3: Resolution of various SLM's. 
156 / Critical Reviews Vol. CR74

Optically addressed SLM's have a higher resolution compared with electrically addressed SLM's.

\section{IMPLEMENTATION OVERVIEW}

The SLM has always been the weakest part among the components of an optical processor. The poor performance and high cost of SLM's have delayed the fabrication of a practical optical processor. As shown in section 2, the situation is now different, because the SLM's used in video projectors are now available at low cost and their characteristics are compatible with the constraints of optical information processing. In this section, we will present some uses of SLM's for DOE's display. This review is not exhaustive but should show the evolution of the use of SLM's for dynamic DOE's with the progress of SLM technology. There are many different SLM's as shown in section 2, but all of them are not adapted for DOE's. An SLM displaying a DOE must transform an incoming wavefront into another wavefront with the desired amplitude and phase. Therefore, it is very important to precisely know the SLM transfer function both in its amplitude and in its phase.

\subsection{The evolution of the SLM's used in video projectors}

Since they were easily available and also the cheapest, the SLM's primarily designed for video projectors were used by a large number of researchers in optical processing.

Around 1980, Hughes Corporation developed an optically addressed SLM for large screen display and this SLM was used in several optical processors. The global performance of this SLM was good, but it was relatively expensive $(\$ 25 \mathrm{~K})$.

Then, about 1985, small Liquid Crystal TV sets were commercialized at a low price. Typically the screen was a matrix of $120 \times 120$ cells of liquid crystal and each pixel was $400 \times 327 \mu \mathrm{m}$. The screen was addressed with a video signal. The LCTV's were widely used, but their characteristics were very poor and not well adapted to optical processing constraints. In particular, the phase was not uniform and had to be corrected by a liquid gate.

In 1990 , the first video projectors were commercialized at a reasonable price. These projectors use $3 \mathrm{LCD}$ matrices, one for each color (red, green, blue). For example in the Epson Crystal Image Video Projector, the SLM's have a resolution of $320 \times 220$ pixels, a pixel pitch of $80 \times 90 \mu \mathrm{m}$ and a pixel size of $55 \times 60 \mu \mathrm{m}$. In many projectors the addressing signal is a video signal. A large number of research groups working in the field of optical information processing dismantled video projectors and used the 3 LCD panels as SLM's. There were many problems; for example it was impossible to address the individual cells with the classic video signal.

Now, the situation is much better, SLM's used in video projectors can now be bought separately; they have a VGA (640x480 pixels), SVGA (600x800) and XGA (1024x768) resolution and can be directly connected to a $\mathrm{PC}$ instead of the regular screen. The pixels are also smaller. For the VGA2 and SVGA commercially available from CRL the pixel pitch is respectively $42 \times 42 \mu \mathrm{m}$ and $33 \times 33 \mu \mathrm{m}$ and the pixel size is respectively $35 \times 33$ $\mu \mathrm{m}$ and $26 \times 24 \mu \mathrm{m}$. These SLM's are available at a reasonable price $(\$ 4 \mathrm{~K})$. Some problems still remain since it is difficult, for example, to control the electronic signals sent to the SLM; most of the time the only adjustments that can be made by the user are brightness and contrast. To know the transfer function of one SLM, the user needs to measure it. 


\subsection{Characterization methods}

In order to correctly display a DOE onto a SLM, it is important to know its characteristics in amplitude and in phase. The characterization methods for TN-LC SLM have been extensively studied. The amplitude characterization presents no major difficulty ${ }^{19}$. For the phase characterization, there are two basic methods. With the first method, the SLM is placed into a Mach-Zehnder interferometer ${ }^{20,21}$. Two different gray levels are displayed on the SLM (one of them is a fixed reference) and the analysis of the displacement of the fringes at the output of the interferometer will give the phase of the SLM in relation with the gray level. The problems of this method are related to the stability of the interferometer and also to the difficulty of measuring the displacement of the fringes due to the noise. Neto et al. ${ }^{6}$ replaced the Mach-Zehnder with a wedge shear plate interferometer which is simple, stable and easy to align.

The second method is based on the measurement of the intensities of the diffraction orders of different patterns displayed or imaged on the SLM. The Young pinholes ${ }^{22}$ were imaged on the SLM and the Ronchi gratings were imaged ${ }^{23}$ or written directly on the $\mathrm{SLM}^{8,24}$. The basic method proposed by Zhang et al. ${ }^{24}$ is valid for the phase mostly mode. An extended version of this method has been presented by Laude ${ }^{8}$; it is based on the Jones Matrix as proposed by Yamauchi ${ }^{25}$.

\subsection{Applications of SLM's for CGH's}

\subsubsection{Use of an Optically Addressed SLM}

Optically addressed SLM's offer the advantage of a higher resolution and smaller pixel size compared with EASLM's. The writing beam carries the optical image of the CGH. In 1990, Fracasso et al. $^{26}$ used a bistable optically addressed ferroelectric liquid-crystal spatial light modulator to record binary CGH's. For the experiment, the CGH was displayed on a photographic plate, and then imaged onto the OASLM. For real time applications, the CGH could have been displayed on a high resolution CRT. The results showed no degradation of the reconstruction of CGH written on the OASLM.

In 1994, Kirk et al. ${ }^{27,28}$ implemented reconfigurable Fourier CGH's by reducing a binary CGH image from an EASLM onto a phase only OASLM. The OASLM was the PAL SLM from Hamamatsu Photonics K.K. ${ }^{10}$. The CGH's were used in an optoelectronic cellular processing system with a reconfigurable holographic interconnects. Simulated annealing and modified error diffusion algorithms were used.

\subsubsection{Use of a Liquid Crystal TV set}

Due to their low price $(\$ 100)$, LCTV's attracted a lot of attention and were inserted into optical information processing systems. Such LCTV's were mostly used in the input plane. Yu et al. ${ }^{29}$ for example used the LCTV in a joint transform correlator. In their paper, Lu and Saleh ${ }^{21}$ characterize a LCTV(Realistic Model 16-151) and give a list of references of applications of LCTV's.

The optical quality of such an LCTV was poor, and so it was not possible to use them directly for dynamic CGH's. To solve this problem, Mok et al. ${ }^{30}$ inserted the LCTV into a liquid gate, displayed real time CGH's onto it and obtained a relatively good optical reconstruction. 
158 / Critical Reviews Vol. CR74

\subsubsection{Use of video Liquid Crystal display}

This type of liquid crystal SLM extracted from a video projector was extensively used and studied. Kirsch et al. ${ }^{20}$ and Laude et al. ${ }^{19}$ gave a complete characterization of the SLM's from an Epson video projector. Since the characteristics were slightly different, from one SLM to another, it is necessary, when using a new SLM, to perform another characterization .

Neto et al. ${ }^{6}$ have shown that by adjusting the angles of the polarizer and of the analyzer that are before and after the SLM, it is possible to obtain a specific operating curve and even to operate in a phase mostly mode. They have computed CGH's with an iterative method based on the Gerchberg-Saxton ${ }^{31}$ algorithm that uses the operating curve of the SLM as a constraint in the hologram plane. They also cascaded two SLM's ${ }^{32}$, one operating in the amplitude mostly mode and the other operating in the phase mostly mode with a $2 \pi$ phase modulation range. Again, the computation of the full complex CGH includes the constraints of the two SLM's.

Amako et al..$^{33,34}$ also displayed CGH's onto two cascaded SLM's, onto a TN-LCD for amplitude modulation and onto a specially designed electrically controlled birefringent device for phase modulation.

Juday et al. ${ }^{7}$ have computed CGH's optimized for the SLM's characteristics and have developed an electronic circuit that allowed addressing pixels individually with a video signal.

\subsubsection{Use of VGA or SVGA display}

In the latest projectors, the SLM's are addressed with a VGA or SVGA signal and so, each pixel can be addressed individually. But since of the resolution of these SLM's is higher, most of the time they are thinner and consequently the maximum phase shift will be less than $2 \pi$ making it difficult to use them for kinoforms that are phase only CGH's. Several authors have already presented applications of such SLM's that are commercially available in a kit including the SLM and the driver.

In 1997, Haist et al. ${ }^{35}$ computed a CGH from a 3-D object consisting of two planes with a specially modified Gerchberg-Saxton algorithm and displayed it on a VGA SLM from CRL. The results were good and since the CGH was a binary amplitude CGH, no optimization using the transfer function of the SLM was necessary. Recently, the same group $^{36}$ used a CGH written onto this SLM to generate dynamic light fields of an arbitrary shape. The application was optical particle trapping.

In 1996, Laude ${ }^{8}$ implemented a programmable lens onto a VGA liquid crystal SLM. He studied the optimization of the $\mathrm{CGH}$ coding and concluded that Euclidean Projection gave a solution near the absolute optimum.

\subsubsection{Other SLM's}

Other types of SLM's were used for implementing dynamic CGH's. But these SLM's are more expensive than the LC SLM's used in video projectors and not so easily available.

In 1992, Rosen et al. ${ }^{37}$ computed binary CGH's for a magneto-optic SLM with a resolution of $128 \times 128$ pixels. The CGH's are computed using an iterative algorithm, and the originality of their approach is that the iterations are performed optically. A binary CGH is displayed on the SLM, the complex image reconstruction is detected and a cost function is computed; the CGH displayed on the SLM is then iteratively modified until 
the cost function is below a given desired minimal value. In this case, the computed CGH was optimized for the characteristics of the SLM.

In 1993, Mc Owan et al. ${ }^{38}$ fabricated a specially designed Liquid Crystal to implement a switchable binary Gabor lens.

A magneto-optic SLM was used to implement Fresnel lenses in 1995 by Davis et al. ${ }^{39}$.

In 1994, Morris et al ${ }^{40}$ proposed to combine a phase SLM with a CGH to implement dynamic optical interconnects. The phase SLM changes the phase of the wavefront that illuminates the CGH. The phase SLM must be specially designed for this approach.

In 1996, Takaki et al. ${ }^{41}$ constructed an incoherent pattern detection processor using a liquid crystal active lens consisting of a lens and a phase EASLM.

Yang et al. $^{42}$ cascaded two binary phase ferroelectric SLM's for optical crossbar interconnects.

\section{OCC SIMULATIONS}

Many implementations of DOE's onto SLM's have already been considered. In this paper, our purpose is to give a general framework rather than to provide a turn-key method. Instead of asking the question: " how can we implement DOE onto SLM's, particularly on TN-LCD's? ", we should ask ourselves "what is the point of implementing DOE onto SLM's, especially TN-LCD's? ".

First, the answer is purely technological: It allows us to implement dynamic reconfigurable DOE's. But another point should been considered : TN-LCD's may also alleviate some of the limitations of classic non reconfigurable devices from the point of view of modulating capabilities, for instance.

To highlight the advantages of implementing DOE's onto TN-LCD's, we use the practical, rigorous framework of optimal trade-off (OT), already applied to this domain ${ }^{43}$. We consider two criteria : the optical efficiency $\eta$ :

$$
\eta=\frac{\sum_{(q, r) \in \mathfrak{I}}|g(q, r)|^{2}}{N . M}
$$

and the normalized amplitude error ERRA:

$$
E R R A=\frac{\sum_{(q, r) \in \mathfrak{I}}[|f(q, r)|-\alpha|g(q, r)|]^{2}}{\sum_{(q, r) \in \mathfrak{I}}|f(q, r)|^{2}}
$$

with

$$
\alpha=\frac{\sum_{(q, r) \in \mathfrak{I}}|f(q, r)| \cdot|g(q, r)|}{\sum_{(q, r) \in \mathfrak{I}}|g(q, r)|^{2}}
$$

where $f(q, r)$ is the desired pattern and $g(q, r)$ is the pattern reconstructed by the hologram. The error is only determined in the signal-reconstruction area $\mathfrak{I}$ and N.M is 
the total number of pixels of the DOE. The coding domain (set of possible values for each pixel) is $D$. Optimal trade-off design is not limited in terms of criteria and if necessary, other criteria can be included in the design operation. In the following, SNR, related to 1/ERRA, may be considered instead of ERRA. Of course, the point will be to maximize SNR or $\eta$, whereas ERRA is to be minimized.

In general, the optimal holograms for the normalized amplitude error ERRA and for the diffraction efficiency $\eta$ are different : in practice, these two criteria are antagonistic and the filter optimizing ERRA shows a very low $\eta$ (and vice-versa). In this context, it has been show $\mathrm{n}^{43}$ that computing an optimal trade-off DOE can significantly improve this situation. By definition, a DOE $d_{0}$ is said to be an optimal trade-off for ERRA and $1 / \eta$ in $D^{N . M}$, if there is no other DOE $d$ that verifies:

$$
\operatorname{ERRA}(d) \leq \operatorname{ERRA}\left(d_{0}\right) \text { and } 1 / \eta(d) \leq 1 / \eta\left(d_{0}\right)
$$

with at least one strict inequality. In practice, it comes down to fix one criterion and optimize the other one.
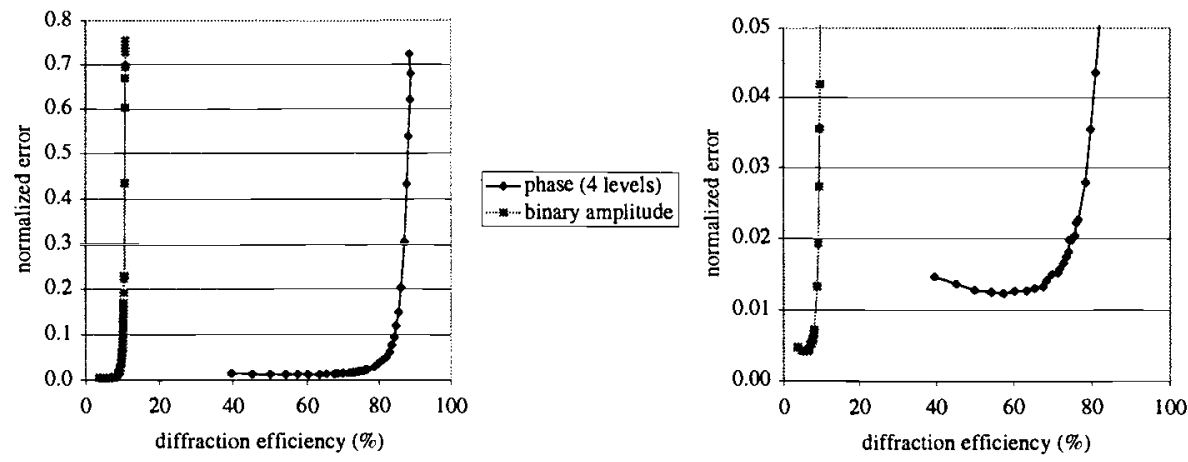

Fig. 4: Example of optimal characteristics curve (OCC) for the binary coding domain (from ref.

${ }^{43}$ ). The view on the right consists of a close-up of the view on the left.

In Fig. 4, a typical locus of points ( $\eta$, ERRA), named optimal characteristics curve (OCC), is reported in the case of two classic coding domains: binary amplitude and 4 levels of phase. We see that the point of optimal trade-off design is to show that a slight degradation in ERRA widely increases $\eta$ (and vice-versa).

The method used in the present paper to compute the DOE's, independently of the optimal-trade-off design, is a multilevel version of the Direct Binary Search (DBS) ${ }^{3}$, a Monte Carlo technique known to provide excellent results but computer intensive. The reason for such a choice is twofold: no method (except simulated annealing algorithm applied to DBS ${ }^{44}$ ) is known to be better than DBS ${ }^{43,45}$ and the adaptation of DBS to the kind of coding domains we consider in this paper is very simple. Nevertheless, the use of other methods such as IFTA ${ }^{1}$, POCS ${ }^{46}$ or HOLOMED ${ }^{7}$ must not be rejected since these methods are known to require much fewer computations than DBS while giving interesting results, sometimes rather close to the ones given by DBS. The classic Euclidean projection, while straightforward and computer inexpensive, is also reported to give results close to the ones provided by $\mathrm{DBS}^{8}$. In any case, as stated earlier, providing another method for implementing DOE's onto SLM's with an arbitrary coding domain is not within the scope of the present paper: the point is rather to decide if it is worth implementing DOE's on SLM's such as TN-LCD's. 
The figures given here concern a large number of simulations, using a set of 10 images with different characteristics. The resolution or space-bandwidth product (SBP) of the images is fixed (32x32 pixels), corresponding to DOE's with $128 \times 128$ pixels. Each point of our OCC's consists of a mean of the criteria obtained for the 10 corresponding DOE's.

In Fig. 4, we briefly recall results already obtained with classic coding domains ${ }^{43}$. Binary amplitude holograms can be displayed on non-reconfigurable devices (photographic emulsion) or SLM's, such as TN-LCD's, FLC-LCD's or MO-LCD's. They usually provide poor diffraction efficiency and an acceptable SNR, while the 4level phase domain allows high diffraction efficiency and poor SNR.

In Fig. 5, we report further simulations concerning eight-level pure amplitude DOE's (it should be reminded that no commercial SLM is able to produce the corresponding coding domain (a similar one is depicted Fig. $1 \mathrm{~g}$ ), and that fabricating such an element still proves expensive. Only one company, Canyon Materials (from San Diego, Ca.), is reported to fabricate multilevel amplitude-only masks. So these simulations just give us a starting point for the discussion. In general, such DOE's show large SNR and poor diffraction efficiency. A classic alternative consists in using phase-only DOE's coded onto eight levels (Fig. 1h). Such elements prove more attractive since they can be fabricated on fixed masks (glass substrates, photopolymers,...) or coded onto TN-LCD's in phase-only (or rather phase-mostly) mode ${ }^{8}$. In this case, since no energy is absorbed by the element and since the conjugated order and the zero-order of the reconstruction can be considerably reduced, great diffraction efficiency is obtained, but on the other hand SNR proves poor (further simulations show that increasing the number of levels can increase the SNR, but cannot compensate for the lack of non-unity amplitude levels).
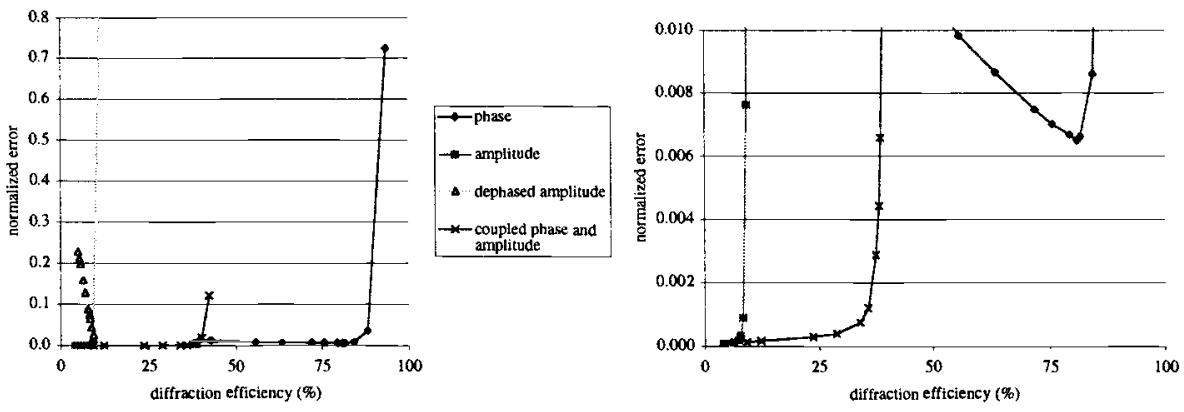

Fig. 5: optimal characteristics curve (OCC) for various coding domains quantized on 8 levels (include amplitude-only, phase-only, amplitude displayed as is, i.e. with a 'parasitic' phase, and the ideal $360^{\circ}$ spiral coding domain described in Fig. 1i)(a). The graph on the right (b) consists of a zoom in a region of particular interest of the OCC. In this close-up, the curve related to the 'dephased amplitude modulation' does not appear since the lowest error value for this modulation is above 0.025 .

Another set of figures reported Fig. 5 concerns the classic (non optimized) implementation of amplitude-only DOE's on an SLM showing an amplitude-phase coupling. This kind of implementation is not theoretically suitable since it does not take account of the dephasing introduced by the SLM (however, some opticians refuse to explicitly take this dephasing into account: so, in this case, we will call it parasitic). The simulations finally prove that this implementation should be avoided as much as 
possible, from a pragmatic point of view : it does not give good results. Efficiency remains low while SNR is strongly degraded.

The simulations concerning improved implementations of DOE's onto a coupled phaseamplitude domain (we chose an ideal spiral with a $360^{\circ}$ maximum dephasing, see Fig. 1i) are also reported Fig. 5. They give three reasons for implementing DOE's on such a domain : (i) this technique allows the use of a dynamic device to write DOE's (ii) it obtains interesting performance (compared with the classic one described above) (iii) it allows us to investigate a region of the locii of trade-offs that was not attainable with other implementations. In particular, the figures provided show that coupled amplitude and phase DOE's behave in an intermediate way between amplitude-only and phaseonly elements. They show good SNR and good diffraction efficiency.

\section{EXPERIMENTAL VALIDATION}

We tried to implement DOE's onto a recently released high SBP (SVGA, $800 \times 600$ pixels) TN-LCD from CRL. Unfortunately, these devices show a limited dephasing capability (less than $3 \pi / 2$ for a red He-Ne ray). It seems that the more recent, the thinner they are. It can be noticed that, earlier this year, CRL released an XGA $(1024 \times 768)$ device ${ }^{47}$ whose phase modulating capabilities have not yet been reported. Nevertheless, we will show in the following that using SLM's with such low dephasing ability is possible for displaying coupled phase and amplitude DOE's.

Fig. 6 reports the coding domains of the SLM obtained for various configurations of the polarizer and analyzer denoted $\left(\psi_{\mathrm{p}}, \psi_{\mathrm{a}}\right)$. As expected, each of them shows, a phase modulation coupled to the amplitude modulation. The same experiments on similar devices have shown that a phase-only (or at least, phase-mostly) mode is also possible, but we are interested in coupled phase and amplitude modes in order to implement DOE's with better SNR, as planned in Section 4.

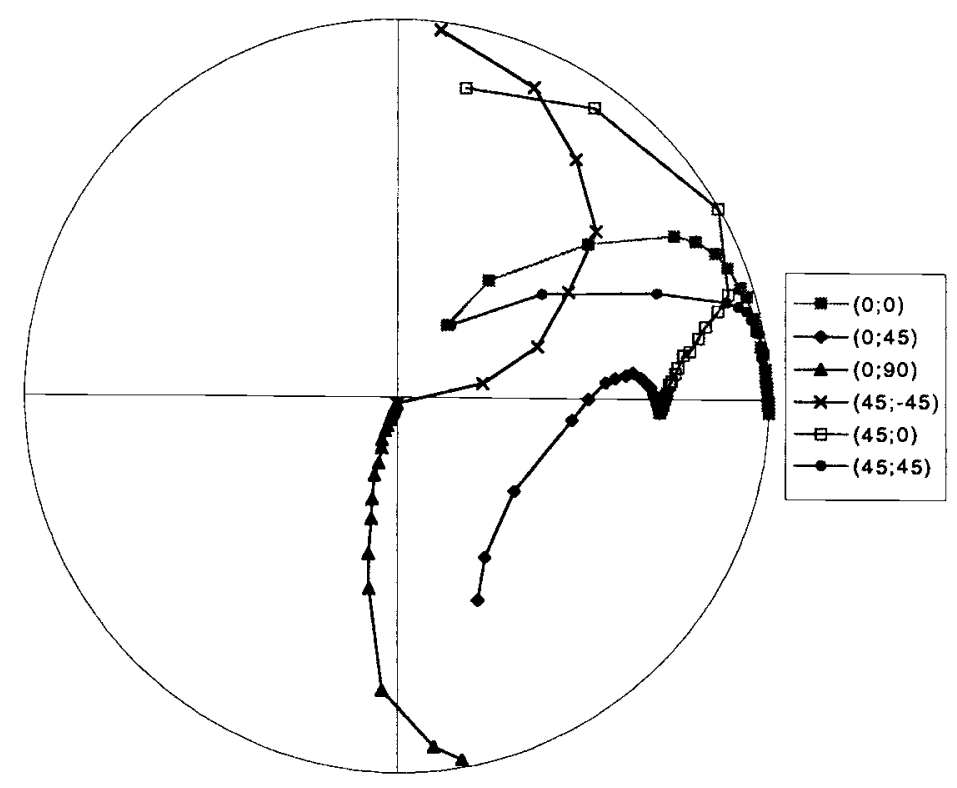

Fig. 6: Various coding domains available for the CRL SVGA TN-LCD 
The configuration we chose is the best compromise between contrast and phase ranging (in this case $\left.83^{\circ}\right)$, corresponding to $\left(\psi_{\mathrm{p}}=45^{\circ}, \psi_{\mathrm{a}}=-45^{\circ}\right)$. The coding domains presented Fig. 6 show low dephasing capabilities. This is only due to the settings of brightness and contrast of the SLM electronics. Larger dephasings can be produced: possible figures are similar to the ones of another device from CRL, VGA2, used by many opticians. Anyway, such a small dephasing will help us to demonstrate that SLM's with a low dephasing capability can be used as coupled phase and amplitude modulators.

Before performing the implementation, we decided to simulate the optical reconstructions (Fig. 7). The first view (Fig. 7a) corresponds to the classic binary implementation. The second view (Fig. 7b) corresponds to the amplitude-only domain quantized on eight levels. The results improve (the resemblance, at least), but the reader should remember that this implementation is not possible at the moment with currently available SLM's. Two implementations of the pure 8-level amplitude DOE 'as is', when the 'parasitic' phase produced by the TN-SLM is not taken into account during the DOE design, are then simulated. Fig. $7 \mathrm{c}$ reports the simulation for an ideal $2 \pi$ spiral, Fig. $7 \mathrm{~d}$ the simulation for the coding domain we experimentally characterized earlier with our $\operatorname{SLM}\left(\psi_{\mathrm{p}}=45^{\circ}, \psi_{\mathrm{a}}=-45^{\circ}\right)$. As already reported in Section 4 , the implementation onto the ideal $2 \pi$ spiral provides poor results and should be avoided. But surprisingly, the implementation onto our experimental coding domain is not so bad, even if it has not been, in this case, taken into account in the DOE design. The design and the implementation onto an ideal $2 \pi$ spiral is simulated Fig. $7 \mathrm{e}$. We clearly see that such an implementation, as explained in Section 4, provides good resemblance (high SNR), and the conjugated order has disappeared. Unfortunately, this implementation is not possible onto most SLM's because their possible maximum dephasing is less than $2 \pi$. Finally, we simulated the implementation onto the coding domain we can address on our SVGA TN-SLM in the configuration $\left(\psi_{\mathrm{p}}=45^{\circ}, \psi_{\mathrm{a}}=-45^{\circ}\right)$, described Fig. 6 . The resemblance seems as good as in the previous case. The only difference is in the conjugated order that can be observed, contrary to the previous case, although it is much weaker than in the case of a binary or amplitude-only modulation. The values of the two criteria are reported in Table 2. 


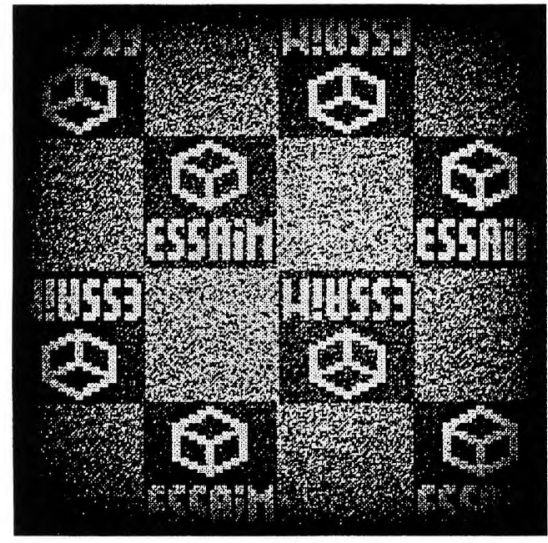

(a)

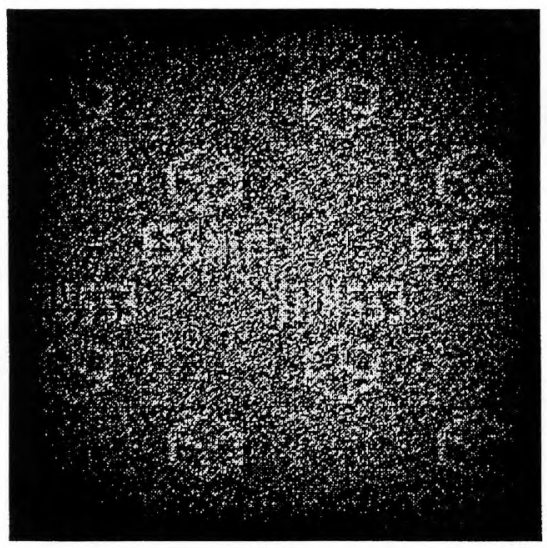

(c)

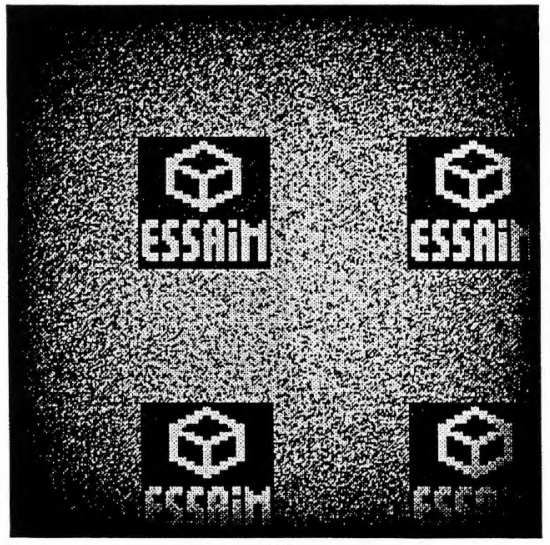

(e)

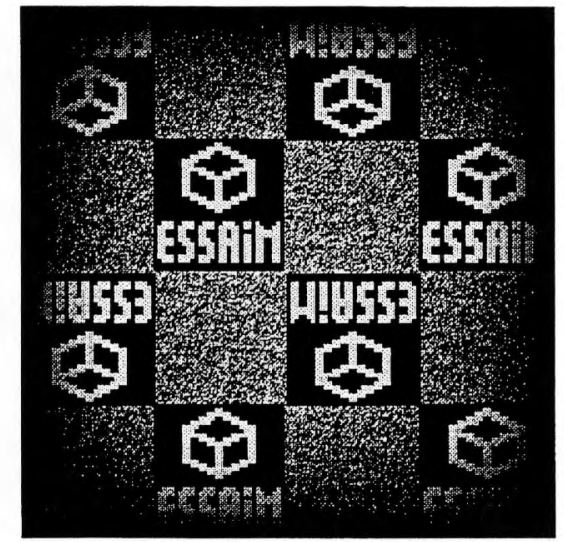

(b)

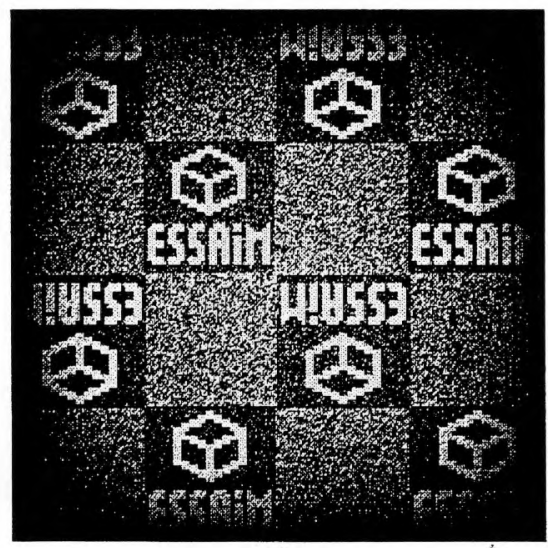

(d)

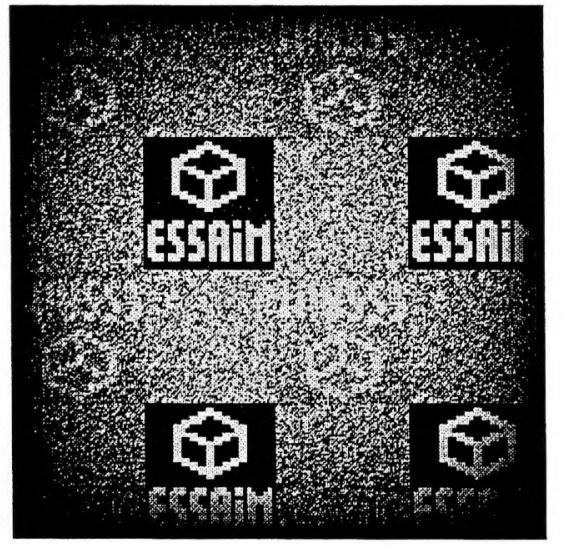

(f)

Fig. 7: Simulation of reconstructions provided by various implementations of DOE's: binary amplitude (a), 8-level amplitude only that is, at the moment, only possible in simulations (b), 8level amplitude only displayed with a parasitic phase ranging from $0^{\circ}$ to $360^{\circ}$ (c), ), 8-level amplitude only displayed with a parasitic phase ranging from $0^{\circ}$ to $83^{\circ}$ (d), 8-level ideal $360^{\circ}$ spiral (e), 8-level experimental spiral with $\left(45^{\circ},-45^{\circ}\right)$ configuration (f). 
A simple validation has been done, with three experiments corresponding to all the possible implementations among the implementations simulated. The first implementation (Fig. 8a) corresponds to a binary DOE, the second one (Fig. 8b) to an 8level amplitude DOE displayed as it is, with the coupled phase modulation not taken into account during the design, and the third one (Fig. 8c) to a phase-amplitude hologram for which the coupled phase has been taken into account in the DOE design. The values of both criteria have been reported in Table 2. All three implementations succeed in reconstructing the desired pattern. The binary implementation (Fig. 8a) gives good reconstruction, with a high contrast in the region of interest. The zero order is quite strong. The direct implementation of the 8-level amplitude-only DOE onto the TNSLM is less good than the previous one (contrast is bad), whereas simulations were close (Fig. 7a and 7d and Table 2, SNR).

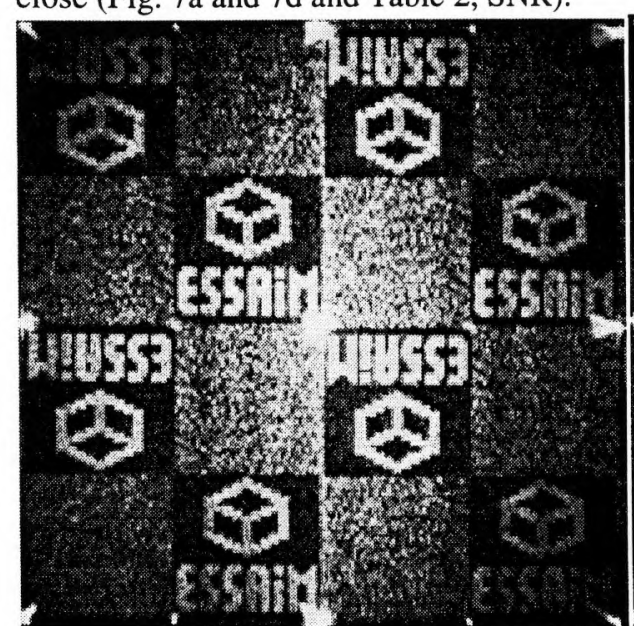

(a)

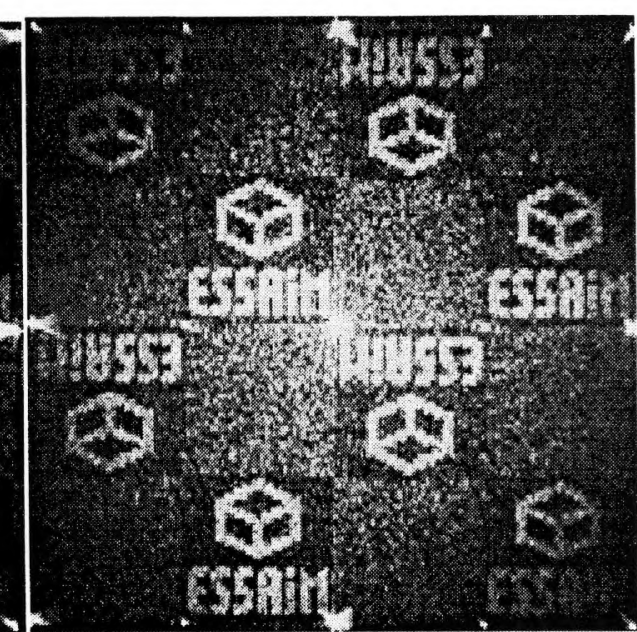

(b)

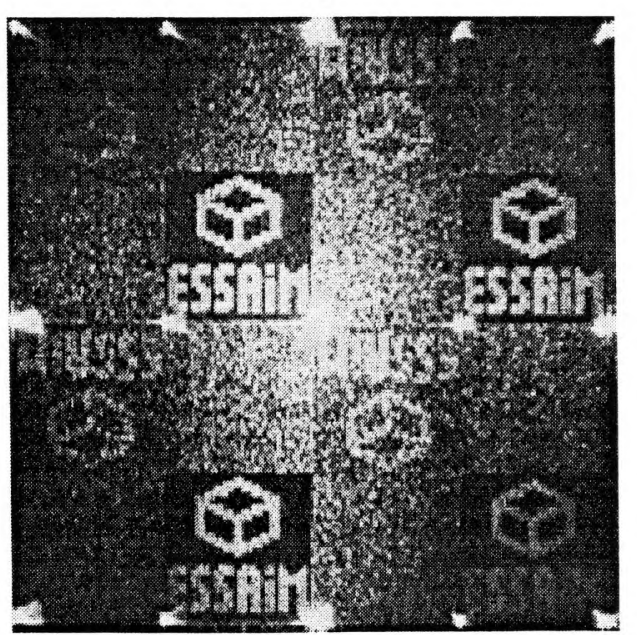

(c)

Fig. 8: Reconstruction obtained from a binary DOE (a), an eight-level amplitude-only DOE displayed on a TN-SLM (b) and an eight-level optimized phase-amplitude DOE (c)

Finally, the implementation of the 8-level coupled phase and amplitude DOE specially designed for the implementation onto the TN-SLM is interesting. Contrast is not so good 
166 / Critical Reviews Vol. CR74

as in the binary case, but the region of interest is uniform and the conjugated order vanishes a little. Nevertheless, as reported in Table 2, the diffraction efficiency figures are disappointing since in this case, they should be twice as large as in the binary case, and actually they are almost twice as small. Three explanations can be considered: the characterization of the spiral coding domain was imperfect (while, it is much better because it is much simpler in the binary case), the electronics may not reproduce 8 gray levels (the data sheet from CRL is not clear) and we considered a global behavior of the SLM whereas such TN-LCD's are reported to show large variations between their pixels $^{48}$

In Table 2, we summarize the various results obtained in simulations and experiments. The reader should remember that the purpose of the present paper is not to provide an efficient technique from the computational point of view: the use of DBS implies huge computing time, which may be alleviated with an adapted version of IFTA, as reported in ref. ${ }^{49}$.

\begin{tabular}{|c|c|c|c|c|}
\hline & \multicolumn{3}{|c|}{ simulations } & experiments \\
\hline coding & $\begin{array}{l}\text { diffraction } \\
\text { efficiency }\end{array}$ & SNR & computation time & energy in order +1 \\
\hline binary & $6.61 \%$ & 16 & $15 \mathrm{~min}$ & $2.3 \mu \mathrm{W} / \mathrm{cm}^{2}$ \\
\hline ideal amplitude 8 & $3.87 \%$ & 253 & $17 \mathrm{~min}$ & \\
\hline $\begin{array}{c}\text { amplitude } 8 \\
\text { displayed on } 2 \pi \\
\text { spiral TN-SLM }\end{array}$ & $28.61 \%$ & 1 & $17 \mathrm{~min}$ & \\
\hline $\begin{array}{c}\text { amplitude } 8 \\
\text { displayed on TN- } \\
\text { SLM } \\
\end{array}$ & $4.71 \%$ & 12 & $17 \min$ & $1.2 \mu \mathrm{W} / \mathrm{cm}^{2}$ \\
\hline ideal $2 \pi$ spiral 8 & $10.95 \%$ & 152 & $11 \mathrm{~h} 01 \mathrm{~min}$ & \\
\hline $\begin{array}{c}\text { spiral } 8 \text { on our } \\
\text { SVGA TN-SLM }\end{array}$ & $3.00 \%$ & 216 & $11 \mathrm{~h} 36 \mathrm{~min}$ & $1.2 \mu \mathrm{W} / \mathrm{cm}^{2}$ \\
\hline
\end{tabular}

Table 2: results obtained in simulation and experiments for various coding domains. The reader must remember that when a pure amplitude hologram is displayed on a TN-SLM, a coupled phase modulation, inherent to the TN-SLM, is added, and the results are degraded

\section{CONCLUSIONS}

In this paper, a review of the implementation of DOE's onto SLM's was presented. We particularly focused on SLM's with modulating capabilities other than binary, e.g. TNSLM's which provide phase-mostly or coupled phase-amplitude modulation. We showed the point in using this latter mode. Considering the general framework of OT design, we showed that it allowed us to investigate a region of trade-offs that are very different from the ones provided by classic coding domains: while the phase-only mode or the amplitude-only mode provide a good value for only one criterion (resp. diffraction efficiency and SNR) and a poor value for the other, this phase-amplitude modulation has an intermediate behavior which gives acceptable values for both criteria. An experimental validation was also provided. It confirmed that displaying a pure amplitude hologram on a TN-SLM, without taking account of the device characteristics during the design, was the worse solution and that the use of the coupling characteristic phase- 
amplitude of the TN-SLM was a valuable alternative for displaying multilevel DOE's with a high SNR. Nevertheless, from the experimental point of view, the classic easy binary implementation still challenges this optimized multilevel implementation. This latter would probably prove better with an SLM with an improved electronic drive board and with pixels that have the same characteristics and behave exactly the same way.

\section{ACKNOWLEDGMENTS}

The authors thank the Conseil Général du Haut-Rhin for their support.

\section{REFERENCES}

1. F. Wyrowski and O. Bryngdahl, "Iterative Fourier-transform algorithm applied to computer holography", J. Opt. Soc. Am. A Vol. 5 (7), pp. 1058-1065, 1988.

2. F. Wyrowski, "Iterative quantization of digital amplitude holograms", Appl. Opt. Vol. 28 (18), pp. 3864-3870, 1989.

3. M.A. Seldowitz, J.P. Allebach and D.W. Sweeney, "Synthesis of digital holograms by direct binary search", Appl. Opt. Vol. 26 (14), pp. 2788-2798, 1987.

4. M. Kufner and S. Kufner, "Micro-optics and Lithography", VUBPRESS (1997).

5. J. Turunen and F. Wyrowski, "Diffractive optics for industrial and commercial applications " (Akademie Verlag, Berlin, 1997).

6. L.G. Neto, D. Roberge and Y. Sheng, "Programmable optical phase-mostly holograms with coupled-mode modulation liquid-crystal television", Appl. Opt. Vol. 34 (11), pp. 1944-1950, 1995.

7. R.D. Juday and J. Knopp, "HOLOMED - An algorithm for computer generated holograms", Optical Pattern Recognition VII, Orlando, D.P. Casasent \& T.-H. Chao ed., SPIE Vol. 2752, pp. 162-172, 1996.

8. V. Laude, "Twisted-nematic liquid-crystal pixelated active lens", Opt. Commun. Vol. 153 (1,2,3), pp. 134-152, 1998.

9. S.-T. Wu, "Nematic liquid crystal", in Spatial light modulator technology: materials, devices, and applications, Marcel Dekker, Inc., 1-31 (1995).

10. N. Mukohzaka, N. Yoshida, H. Toyoda, Y. Kobayashi and T. Hara, "Diffraction efficiency analysis of a parallel-aligned nematic-liquid-crystal spatial light modulator", Appl. Opt. Vol. 33 (14), pp. 2804-2811, 1994.

11. G. Moddel, "Ferroelectric Liquid Crystal Sapatial Light Modulators", in Spatial light modulator technology: materials, devices, and applications, Marcel Dekker, Inc., 287-359 (1995).

12. W.E. Ross and J.A. Davis, "The magneto-optic spatial light modulator", in Spatial light modulator technology: materials, devices, and applications, Marcel Dekker, Inc., 361-390 (1994).

13. S.D. Lindell and D.L. Flannery, "Experimental investigation of transform ratio ternary phase-amplitude filters for improved correlation discrimination", Opt. Eng. Vol. 29 (9), pp. 1044-1051, 1990. 
14. D.R. Pape and L.J. Hornbeck, "Characteristics of the deformable mirror device for optical information processing", Opt. Eng. Vol. 22 (6), pp. 675-681, 1983.

15. J.M. Florence and R.D. Juday, "Full-complex spatial filtering with a phase mostly DMD", Wave Propagation and Scattering in Varied Media II, SPIE Vol. 1558, pp. 487-498, 1991.

16. T.G. Mc Donald and L.A. Yoder, "Digital micromirror devices make projection display", Las. Foc. World Vol. 33 (8), pp. S5-S8, 1997.

17. A.L. Lentine, "Self-electro-optic effect devices for optical information processing", in Optical Computing Hardware, Academic Press, Inc., 45-72 (1994).

18. U. Efron and G. Livescu, "Multiple quantum well spatial light modulators", in Spatial light modulator technology: materials, devices, and applications, Marcel Dekker, Inc., 217-286 (1995).

19. V. Laude, S. Mazé, P. Chavel and P. Réfrégier, "Amplitude and phase coding measurements of a liquid crystal television", Opt. Commun. Vol. $103(1,2)$, pp. 3338, 1993.

20. J.C. Kirsch, D.A. Gregory, M.W. Thie and K.J. Jones, "Modulation characteristics of the Epson liquid crystal television", Opt. Eng. Vol. 31 (5), pp. 963-970, 1992.

21. K. Lu and B. Saleh, "Theory and design of the liquid crystal TV as an optical spatial phase modulator", Opt. Eng. Vol. 29 (3), pp. 240-246, 1990.

22. A. Bergeron, et al., "Phase calibration and applications of a liquid-crystal spatial light modulator", Appl. Opt. Vol. 34 (23), pp. 5133-5139, 1995.

23. C. Soutar and S.E. Monroe, "Measurement of the complex transmittance of the Epson liquid crystal television", Opt. Eng. Vol. 33 (4), pp. 1061-1068, 1994.

24. Z. Zhang, G. Lu and F.T.S. Yu, "Simple method for measuring phase modulation in liquid crystal television", Opt. Eng. Vol. 33 (9), pp. 3018-3022, 1994.

25. M. Yamauchi and T. Eiju, "Optimization of twisted nematic liquid crystal panels for spatial light phase modulation", Opt. Commun. Vol. 115, pp. 19-25, 1995.

26. B. Fracasso, J.L. de Bougrenet de la Tocnaye and P. Ambs, "Recording reconfigurable binary computer-generated holograms on bistable optically addressed ferroelectric liquid-crystal spatial light modulators", Opt. Lett. Vol. 15 (24), pp. 1473-1475, 1990.

27. A. Kirk, T. Tabata and M. Ishikawa, "Design of an optoelectronic cellular processing system with a reconfigurable holographic interconnect", Appl. Opt. Vol. 33 (8), pp. 1629-1639, 1994.

28. A. Kirk, T. Tabata and M. Ishikawa, "Reconfigurable computer generated holograms", Opt. Commun. Vol. 105 (5,6), pp. 302-308, 1994.

29. F.T.S. Yu, S. Jutamulia, T.W. Lin and D.A. Gregory, "Adaptative real-time pattern recognition using a liquid crystal TV based joint transform correlator", Appl. Opt. Vol. 26 (8), pp. 1370-1372, 1987.

30. F. Mok, J. Diep, H.-K. Liu and D. Psaltis, "Real-time computer generated hologram by means of liquid-crystal television spatial light modulator", Opt. Lett. Vol. 11 (11), pp. 748-750, 1986. 
31. R.W. Gerchberg and W.O. Saxton, "A practical algorithm for the determination of phase from image and diffraction plane pictures.", Optik Vol. 35 (2), pp. 237-246, 1972.

32. L.G. Neto, D. Roberge and Y. Sheng, "Full range continuous complex modulation using two led-mode liquid crystal televisions", Appl. Opt. Vol. 35, pp. 4567-4576, 1996.

33. J. Amako, H. Miura and T. Sonehara, "Wave-front control using liquid-crystal devices", Appl. Opt. Vol. 32 (23), pp. 4323-4329, 1993.

34. J. Amako and T. Sonehara, "Kinoform using an electrically controlled birefringent liquid-crystal spatial light modulator", Appl. Opt. Vol. 30 (32), pp. 4622-4628, 1991.

35. T. Haist, M. Schönleber and H.J. Tiziani, "Computer-generated holograms from 3D-objects written on twisted-nematic liquid crystal displays", Opt. Commun. Vol. 140, pp. 299-308, 1997.

36. M. Reicherter, T. Haist, E.U. Wagemann and H.J. Tiziani, "Optical particule trapping with computer-generated holograms written on a liquid-crystal display", Opt. Lett. Vol. 24 (9), pp. 608-610, 1999.

37. J. Rosen, L. Shiv, J. Stein and J. Shamir, "Electro-optic hologram generation on spatial light modulators", J. Opt. Soc. Am. A Vol. 9 (7), pp. 1159-1166, 1992.

38. P.W. McOwan, M.S. Gordon and W.J. Hossack, "A switchable liquid crystal binary Gabor lens", Opt. Commun. Vol. 103 (3,4), pp. 189-193, 1993.

39. J.A. Davis, A.M. Field and D.M. Cottrell, "Fractional pixel translations of Fresnel lenses written onto spatial light modulators", Appl. Opt. Vol. 34 (5), pp. 790-792, 1995.

40. J. Morris and M. Feldman, "Reconfigurable optical interconnects by a combined computer-generated hologram and a spatial light modulator method", Appl. Opt. Vol. 33 (17), pp. 3683-3694, 1994.

41. Y. Takaki, K. Ishida, Y. Kume and H. Ohzu, "Incoherent pattern detection using a liquid-crystal active lens", Appl. Opt. Vol. 35 (17), pp. 3134-3140, 1996.

42. G.G. Yang and S.E. Broomfield, "programmable wavefront generation using two binary phase spatial light modulators", Opt. Commun. Vol. 124, pp. 345-353, 1996.

43. L. Legeard, P. Réfrégier and P. Ambs, "Multicriteria optimality for iterative encoding of computer generated holograms", Appl. Opt. Vol. 36 (29), pp. 7444$7449,1997$.

44. A.G. Kirk and T.J. Hall, "Design of binary computer generated holograms by simulated annealing: coding densityand reconstruction error", Opt. Commun. Vol. 94, pp. 491-496, 1994.

45. J.P. Allebach and D.W. Sweeney, "Iterative approaches to computer generated holography", Computer-Generated Holography II, S.H. Lee ed., SPIE Vol. 884, pp. $2-9,1988$.

46. A. Levi and H. Stark, "Image restoration by the method of generalized projections with application to restoration from magnitude", J. Opt. Soc. Am. A Vol. 1 (9), pp. 932-943, 1984. 
170 / Critical Reviews Vol. CR74

47. CRL, "XGA1", advertisement, http://www.crl.co.uk/wcr/liquidsun/pro_xg1.htm (1999).

48. C. Soutar, S.E. Monroe, Jr. and J. Knopp, "Measurement of the complex transmittance of the Epson liquid crystal television", Opt. Eng. Vol. 33 (4), pp. 1061-1068, 1994.

49. L. Bigué and P. Ambs, "Filter Implementation technique for multicriteria characterization of coding domains in the joint transform correlator", Appl. Opt. Vol. 38 (20), 1999. 